\title{
Adaptive Multiplicative Noise Removal Model Using Pixel Related Smoothing Term
}

\author{
Yifen Dong*
}

Shandong Vocational College of Economics and Business, Weifang, China

\begin{abstract}
Multiplicative noise removal problems have attracted much attention in recent years. In this paper, we propose a new adaptive multiplicative noise removal algorithm based on variational method. By analysis the shortcoming of EulerLagrange equation, we find that these traditional variational models are not fitted for multiplicative noise very well. The amount of multiplicative noise is relative with the pixel value. That is to say, areas with large pixel value should be smoothed much than areas with small pixel value. So we modified the Euler-Lagrange equation by changing the balance parameter to the pixel related one, then we deduce our modified energy equation. The balance of fidelity term and regularization term in our changed model can be changed in different areas with different gray value. Thus in iterating procedure, our method can change the degree of noise removing in different areas with different noise level adaptively. It can also preserve the edges and remove the noise very well. The results show the outperforming effect of our method.
\end{abstract}

Keywords: Image denoising, multiplicative noise, variational method.

\section{INTRODUCTION}

Multiplicative noise often appeared in many screens such as medical images and aerial images. The noise intensity of the image related with the image gray value. In this paper, we concern with the multiplicative noise removing problem. The equation depicts an image containing multiplicative noise can be summed as: $f=u+u^{*} v$, where $f$ is the noise polluted image, $u$ is the original image $v$ is the multiplicative noise. Our goal is then to recover $u$ from the noised image data $f$. There are many variational methods [1-4] devoted for the removal of multiplicative noise removal. The famous RLO method [1] is the first attempt for multiplicative noise removal using variational method. From then on, many methods were proposed for different usage. AA model [2] was deduced by using MAP estimator, the model fits for the multiplicative noise with Rayleigh distribution. A strictly convex model for multiplicative noise removal is proposed by Huang [3], in this model, the modified total variation regularization is imported in the function for recovering the clear image. L1 norm based fidelity is introduced by Durand [4] and this term is superior for recovering the edges. Setzer [5-8] proposed a multiplicative noise removal model coupling with deblurring. Denis [9] proposed a fast discrete minimization model for the multiplicative noise removal and used it for SAR image denoising. Shi [10] used nonlinear inverse scale space method for multiplicative noise removal and this method is also convex. Huang $[11,12]$ used a learned dictionary method for multiplicative noise removal.

In this paper, traditional noise removal model is incorporated with dictionary learning method.

All the methods mentioned above using variational method to deal with multiplicative noise are focused on fidelity term. None was devoted to the smooth term. In this paper, we calculate the smooth term by change it to fit for multiplicative noise removing. We find that these traditional variational models are not fitted for multiplicative noise very well. The amount of multiplicative noise is relative with the pixel value. That is to say, areas with large pixel value should be smoothed much than areas with small pixel value. So we modified the Euler-Lagrange equation by changing the balance parameter to the pixel related one, then we deduce our modified energy equation. The balance of fidelity term and regularization term in our changed model can be changed in different areas with different gray value.

Our paper was organized as follows. In Section 2, we will introduce some multiplicative noise removal method and propose our method for multiplicative noise removal. Then some numerical examples are shown in Section 3. Section 4 is concluding remarks.

\section{MODIFIED VARIATIONAL MODEL FOR MULTI- PLICATIVE NOISE REMOVING ADAPTIVELY}

From some experiments done by traditional variational method for multiplicative removing, the main drawbacks mainly focus of two points: 1, After denoising there are some spots all over the image. 2, The edge is blurred in almost cases. All the two drawbacks are owing to the inherent character of multiplicative noise. The reason of some spots after noise removing is because in the iteration the gradient in these points is with high value. In areas with high gray 
value, the multiplicative noise is also high, so we need to change the degree of noise removing with the gray value.

Before introducing our model, we recall the RLO model. The RLO model is depicted as (1):

$\min _{u}\left\{E(u)=\lambda \int_{\Omega}|\nabla u| d x d y+\int_{\Omega}\left(\frac{f}{u}-1\right)^{2} d x d y\right\}$

$f$ is noise image and $u$ is the desired original image.

Auber, Aujol [2] used the equation of:

$\min _{u}\left\{E(u)=\lambda \int_{\Omega}|\nabla u| d x d y+\int_{\Omega}\left(\frac{f}{u}+\log u\right) d x d y\right\}$

Setzer, Steidl and Tueber [11] used the equation of:

$\min _{u}\left\{E(u)=\lambda \int_{\Omega}|\nabla u| d x d y+\int_{\Omega}(u-f \log u) d x d y\right\}$

Denis, Tupin, Darbon, Sigelle [9] used the equation of:

$\min _{u}\left\{E(u)=\lambda \int_{\Omega}|\nabla u| d x d y+\int_{\Omega}\left(\frac{1}{2}\left(\frac{f}{u}\right)^{2}+\log u\right) d x d y\right\}$

Shi, Osher [10] used the equation of:

$\min _{u}\left\{E(u)=\lambda \int_{\Omega}|\nabla u| d x d y+\int_{\Omega}\left(a \frac{f}{u}+\frac{b}{2}\left(\frac{f}{u}\right)^{2}+c \log u\right) d x d y\right\}$

All the above model can be rewritten as :

$\min _{u}\left\{E(u)=\lambda \int_{\Omega}|\nabla u| d x d y+\int_{\Omega} H(u) d x d y\right\}$

Where $H(u)$ is the fidelity term. The Euler-Lagrange equation is:

$\lambda \cdot \operatorname{div}\left(\frac{\nabla u}{|\nabla u|}\right)+H^{\prime}(u)=0$

This model only deals with data term but not deals with smooth term. Although the data term was fit for multiplicative noise, the smooth term is not fit for it. Because in different areas with different gray value, the balance parameter of $\lambda$ is same. To change the $\lambda$ for different area, we can modify the descent equation by changing $\lambda$ to $\lambda \cdot u$.

So we can change the above models by modifying the Euler-Lagrange equation. The modified equation is:

$\lambda \cdot u \cdot \operatorname{div}\left(\frac{\nabla u}{|\nabla u|}\right)+H^{\prime}(u)=0$

The above equation equals the following equation:

$\lambda \cdot \operatorname{div}\left(\frac{\nabla u}{|\nabla u|}\right)+\frac{H^{\prime}(u)}{u}=0$

The model is likely the Weberized TV Restoration [7], but the fidelity term is different. We inversely deduce the energy function from the Euler-Lagrange equation, then the variational energy function of the above equation is:

$$
\min _{u}\left\{E(u)=\lambda \int_{\Omega}|\nabla u| d x d y+\int_{\Omega}\left(\int_{\Omega}\left(\frac{H^{\prime}(u)}{u}\right) d u\right) d x d y\right\}
$$

For the explanation of how to deduce the modified model using traditional model, we use RLO [1] model for explanation.

The original Euler-Lagrange equation of (1) is calculated using equation (11),

$\lambda \cdot \operatorname{div}\left(\frac{\nabla u}{|\nabla u|}\right)+2 *\left(\frac{f^{2}}{u^{3}}-\frac{f}{u^{2}}\right)=0$

So the modified RLO method [1] can be changed as Equ. 12

$\lambda \cdot u \cdot \operatorname{div}\left(\frac{\nabla u}{|\nabla u|}\right)+2 \cdot\left(\frac{f^{2}}{u^{3}}-\frac{f}{u^{2}}\right)=0$

$\lambda$ is substituted by $\lambda \cdot u$ for the reason of that the balance parameter can be changed adaptively by the gray value of the image.

Equ. 12 is also equals the following Equ. 13 by divide $u$ :

$\lambda \cdot \operatorname{div}\left(\frac{\nabla u}{|\nabla u|}\right)+2 \cdot \frac{1}{u} \cdot\left(\frac{f^{2}}{u^{3}}-\frac{f}{u^{2}}\right)=0$

From Equ.13 we can get the related energy equation as depicted in Equ.14:

$\hat{u}=\arg \min _{u}\left\{E(u)=\lambda \int_{\Omega}|\nabla u| \mathrm{d} x \mathrm{~d} y+\int_{\Omega}\left(\frac{2 f^{2}}{3 u^{3}}-\frac{f}{u^{2}}\right) \mathrm{d} x \mathrm{~d} y\right\}$

For the same procedure, we can get the other model equations.

Modified Auber, Aujol[2] method can be got:

$\hat{u}=\arg \min _{u}\left\{E(u)=\lambda \int_{\Omega}|\nabla u| \mathrm{d} x \mathrm{~d} y+\int_{\Omega}\left(\frac{f}{2 u^{2}}-\frac{1}{u}\right) \mathrm{d} x \mathrm{~d} y\right\}$

Modified Setzer, Steidl and Tueber[11] method can be got:

$\hat{u}=\arg \min _{u}\left\{E(u)=\lambda \int_{\Omega}|\nabla u| \mathrm{d} x \mathrm{~d} y+\int_{\Omega}\left(\frac{f}{u}+\log u\right) \mathrm{d} x \mathrm{~d} y\right\}$ Eq. (16)

Modified Denis, Tupin, Darbon, Sigelle[9] method can be got:

$$
\hat{u}=\arg \min _{u}\left\{E(u)=\lambda \int_{\Omega}|\nabla u| \mathrm{d} x \mathrm{~d} y+\int_{\Omega}\left(\frac{f^{2}}{3 u^{3}}-\frac{1}{u}\right) \mathrm{d} x \mathrm{~d} y\right\}
$$

From traditional variational method we can get that large $\lambda$ corresponds to smooth large noise while small $\lambda$ can result in little noise removing in the image. If $\lambda$ is selected, the degree of noise removing is fixed all over the image. Because the multiplicative noise is related with the original gray, so $\lambda$ should big in area with high gray value and it should be small with little gray value. We want to find an adaptive regularization term for the purpose of different balance of closeness to data term and filderity term. So we change the regularization term to change the balance for different part of image as depicted in Equ.12. From Equ.12, we find that for a 
fixed $\lambda$, the value of $\lambda \cdot u$ is related with $u$, so our model can deal with multiplicative noise adaptively.

We write the smooth term of various variational model as:

$J(u)=\int_{\Omega}\left(\frac{H^{\prime}(u)}{u}\right) d u$

The modified variation model for multiplicative noise removal is:

$$
E(u)=\lambda \int_{\Omega}|\nabla u| d x d y+\int_{\Omega} J(u) d x d y
$$

We use the split Bregman method for solving the above equation because of its efficiency and accurate. The split bregman method of our model is depiceted in Equ.20.

$$
\begin{aligned}
& E(u, w)=\lambda \int_{\Omega}|w| d x d y+ \\
& \frac{\theta}{2} \int_{\Omega}\left(w-\nabla u-b^{k+1}\right)^{2} d x d y+\int_{\Omega} J(u) d x d y
\end{aligned}
$$

The above equation can be solved through alternatively minimizing $\mathrm{w}$ and $\mathrm{u}$.

For solving $\mathrm{u}$, the related function is:

$$
E(u)=\frac{\theta}{2} \int_{\Omega}\left(w-\nabla u-b^{k+1}\right)^{2} d x d y+\int_{\Omega} J(u) d x d y
$$

From variational method we can get the following EulerLagrange equations:

$\frac{\partial J\left(u^{k+1}\right)}{\partial u}-\theta \nabla \cdot\left(\nabla u^{k+1}-w^{k}+b^{k+1}\right)=0$

For different modified model, $\frac{\partial J(u)}{\partial u}$ has different forms. For modified RLO, $\frac{\partial J(u)}{\partial u}=\frac{2 f^{2}}{3 u^{3}}-\frac{f}{u^{2}}$. For modified Auber, Aujol model $\frac{\partial J(u)}{\partial u}=\frac{f}{2 u^{2}}-\frac{1}{u}$. For modified Setzer, Steidl and Tueber model, $\frac{\partial J(u)}{\partial u}=\frac{f}{u}+\log u$. For modified Denis, Tupin, Darbon, Sigelle model, $\frac{\partial J(u)}{\partial u}=\frac{f^{2}}{3 u^{3}}-\frac{1}{u}$. Submitting $\frac{\partial J(u)}{\partial u}$ of different model to Equ.22, we will get the corresponding discrete equation.

For solving $w$, the energy function is:

$E(w)=\lambda \int_{\Omega}|w| d x d y+\frac{\theta}{2} \int_{\Omega}\left(w-\nabla u-b^{k+1}\right)^{2} d x d y$

we can be computed by Euler-Lagrange equation:

$w=\nabla u+b-\frac{\lambda}{\theta} \frac{w}{|w|}$

$w$ can be computed by generalized soft threshold method.

$w^{k+1}=\operatorname{Max}\left(\left|\nabla u^{k+1}+b^{k+1}\right|-\frac{\lambda}{\theta}, 0\right) \frac{\nabla u^{k+1}+b^{k+1}}{\left|\nabla u^{k+1}+b^{k+1}\right|}, 0 \frac{0}{0}=0$

In iterations of solving the above equations, we set $b^{k+1}=b^{k}+\nabla u^{k}-w^{k}, \quad b^{0}=w^{0}=0, u^{0}=f . \quad$ After some iteration, we will get the minimum of the total function energy. Then we will get the final clear image.

\section{EXPERIMENTAL RESULTS}

In this section, we test the proposed method using different images. The method we compared is the original multiplicative noise removal method for the explanation of the modified term is effective.

For different kind of noise, we use different fidelity term. For judging the performance of the proposed model with the original method, we select several quantitative measurements. They are peak signal-to-noise-ratio (PSNR), signalto-noise-ratio (SNR) and Edge-preservation index (EPI) [5, 6]. PSNR is always used for explanation of the noise removal ability and the EPI is used for explanation of the edge preservation ability. Large PSNR and SNR correspond to the better noise removal ability. Large EPI corresponds to the better edge preservation and the EPI is small than 1 value.

Figs. (1-4) are the results comparison of our method with other famous multiplicative noise removal methods when dealing with large multiplicative noise.

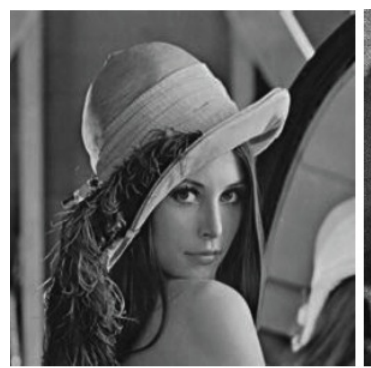

(a)

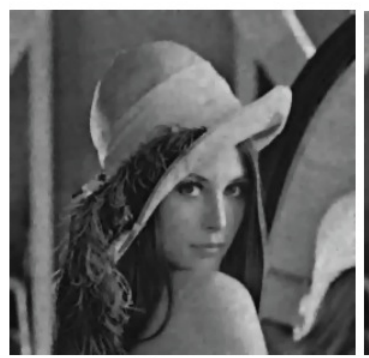

(c)

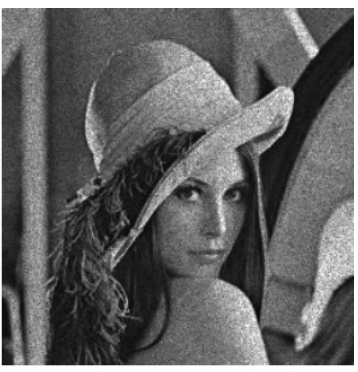

(b)

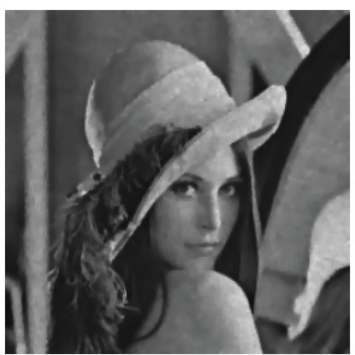

(d)
Fig. (1). Experiment on 'Lena' image. (a) Original image. (b) Polluted by multiplicative noise with Gaussian distribution (mean is 0 , variance is 0.01). (c) Denoised image using RLO method [1] (SNR $=15.7379$, PSNR $=30.3023$, EPI $=0.7912$ ). (d) Denoised image using our method $(\mathrm{SNR}=16.2193, \mathrm{PSNR}=30.9701, \mathrm{EPI}=0.8076)$.

Fig. (1) is the experiment on Lena with Gaussian type of multiplicative noise removing. The RLO method can't deal with noise very well; there are still large noise in flat areas. The proposed method can remove noise very well while the edges are well preserved. The EPI shows the edge preservation ability of our method. The SNR and PSNR show that the noise removal effect of our method is superior to the RLO method. 


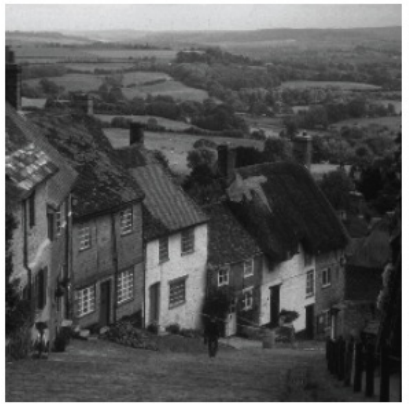

(a)

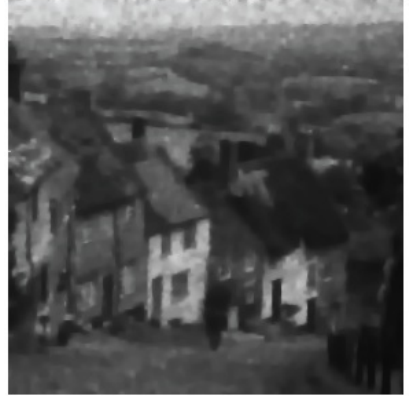

(c)

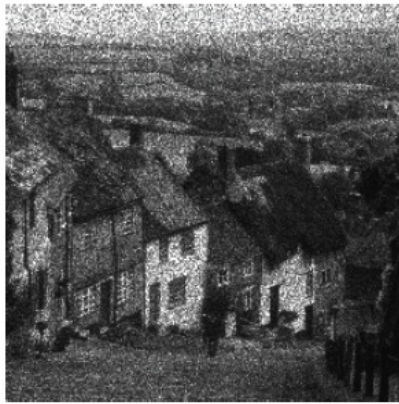

(b)

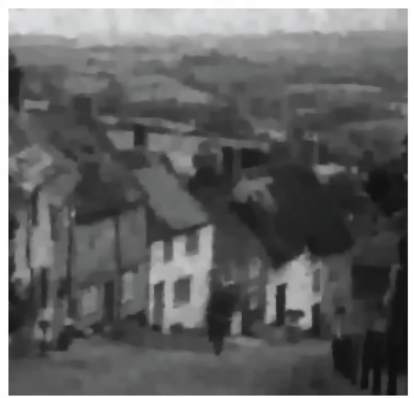

(d)
Fig. (2). Experiment on 'House' image. (a) Original image. (b) Polluted by multiplicative noise with Gamma distribution (mean is 0 , variance is 0.05). (c) Denoised image using AA method [2] (SNR $=11.5039, \mathrm{PSNR}=25.7784, \mathrm{EPI}=0.5509)$. $(\mathbf{d})$ Denoised image using our method $(\mathrm{SNR}=11.9731, \mathrm{PSNR}=25.9935, \mathrm{EPI}=0.6021)$.

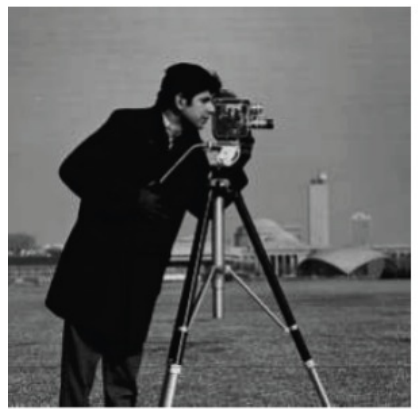

(a)

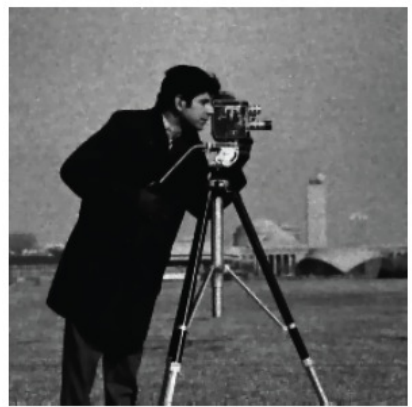

(c)

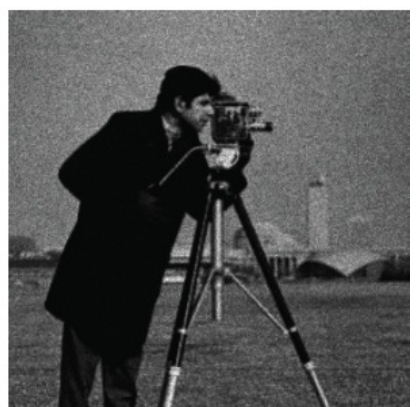

(b)

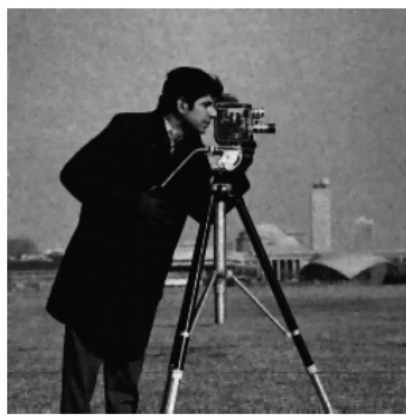

(d)
Fig. (3). Experiment on 'CameraMan' image. (a) Original image. (b) Polluted by multiplicative noise with Poisson distribution(variance is 0.01). (c) Denoised image using Setzer [11] method (SNR $=19.2593$,PSNR $=31.4944, \mathrm{EPI}=0.9039)$. $(\mathbf{d})$ Denoised image using our method $(\mathrm{SNR}=19.9237, \mathrm{PSNR}=32.0139, \mathrm{EPI}=0.9092)$.

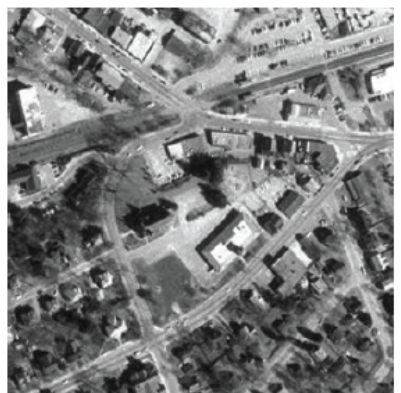

(a)

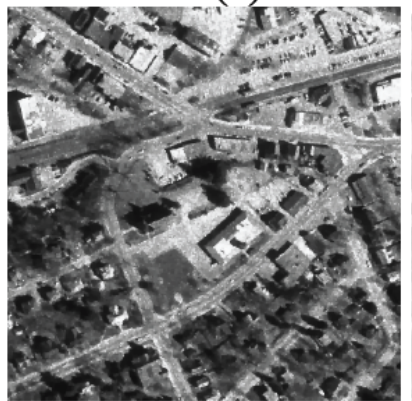

(c)

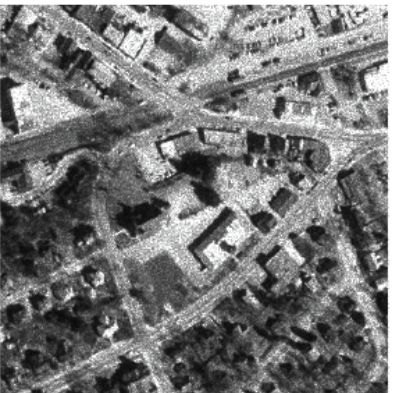

(b)

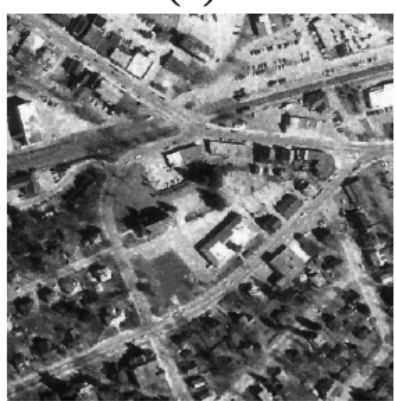

(d)
Fig. (4). Experiment on SAR image. (a) Original image. (b) Polluted by multiplicative noise with Rayleigh distribution(variance is 0.04). (c) Denoised image using Denis method [9] (SNR =13.2056, PSNR $=25.5136$, EPI $=0.7298$ ). (d) Denoised image using our method $(\mathrm{SNR}=13.4107, \mathrm{PSNR}=25.9545, \mathrm{EPI}=0.7426)$

Fig. (2) is the experiment on a 'House' image with Gamma type of multiplicative noise removing. The noise is very large, it is hard to remove. The AA method and our method can both remove this type of multiplicative noise. Experiment shows that our method can get right result, and the large noise is removed while the edges in the image are preserved. The value of SNR, PSNR and EPI using our method are all bigger than AA method, which proves that our method can get better result than AA method.

Fig. (3) is the experiment on 'cameraman' image with Poisson type of multiplicative noise removing. From the experiment, we can see the noise in most sky area and on the buildings faraway is removed. The value of SNR, PSNR and EPI using our method are all bigger than using Setzer method, which proves that our method can get better result than using Setzer method.

Fig. (4) is the experiment on an aerial picture with Rayleigh type of multiplicative noise removing. The edges in the image are all well preserved. The value of SNR, PSNR and EPI using our method are all bigger than using Denis method, which proves that our method can get better result than using Denis method.

The value of objective evaluations about SNR, PSNR and EPI comparison of our method with original methods are listed in figure caption. The SNR, PSNR and EPI got using our method are all large than using the original methods. These data prove that our proposed methods are superior to the original ones. And this phenomenon proves that our methods are better than the original methods. From the 
details in the images, we can also see that our method got right results. This also proves the quantitative indicators.

From visual comparison and the quantization comparison of the above experiments, we can also see that the model proposed in this paper get better results compared with the other methods..

\section{CONCLUSION}

In this paper a new variational model dealing with multiplicative noise is raised. The advantage of our method is that our model can adaptively deal with noise in different areas. The parameter controlling the degree of noise removing is different in different area. Thus our method can get more reasonable results.

\section{CONFLICT OF INTEREST}

The author confirms that this article content has no conflict of interest.

\section{ACKNOWLEDGEMENTS}

This work was supported by National Natural Science Foundation of China (No.61305045) and Natural Science Foundation of Shandong Province (No.ZR2010FQ030).

\section{REFERENCES}

[1] L. Rudin, P. Lions, and S. Osher, "Multiplicative Denoising and Deblurring: Theory and Algorithms," In: Osher S and Paragios N,
Eds, Geometric Level Set Methods in Imaging, Vision and Graphics, Springer, 103-119, 2003.

[2] G. Aubert, and J Aujol, "A variational approach to remove multiplicative noise," SIAM Journal on Applied Mathematics, vol. 68, no. 4, pp. 925-946, 2008.

[3] Y. Huang, M. Ng, and Y. Wen, “A new total variation method for multiplicative noise removal," SIAM Journal on Imaging Sciences, vol. 2, no. 1, pp. 20-40, 2009.

[4] S. Durand, J. Fadili and M. Nikolova, "Multiplicative noise removal using L1 fidelity on frame coefficients," CMLA Report, pp. 0840, 2008.

[5] F. Sattar, L. Floreby, G. Salomonsson, and B. Lovstrom, "Image enhancement based on a nonlinear multiscale method," IEEE Transaction Image Process, vol. 6, no. 9, pp. 888-895, 1997.

[6] R.W. Ives, P. Eichel, and N. Magotra, "A new SAR image quality metric," In: Proceedings of $42^{\text {nd }}$ IEEE Midwest Symposium on Circuits and Systems, vol. 2, pp. 1143-1145, 1999.

[7] S. Jianhong, "Weber's Law and Weberized TV Restoration," CMLA Report, pp. 02-20, 2002.

[8] S. Setzer, G. Steidl and T. Teuber, "Deblurring poissonian images by split bregman techniques," Journal Visual Communication and Image Representation, 2009.

[9] L. Denis, F. Tupin, J. Darbon, and M. Sigelle, "SAR image regularization with fast approximate discrete minimization," IEEE Transaction Image Process, vol. 18, no. 7, pp. 1588-600, 2009.

[10] J. Shi, and S. Osher, "A nonlinear inverse scale space method for a convex multiplicative noise model," SIAM Journal of Imaging Sciences, vol. 1, no.3, pp. 294-321, 2008

[11] Y. Huang, L. Moisan, M.K. Ng, and T. Zeng, "Multiplicative Noise Removal via a Learned Dictionary," IEEE Transactions on Image Processing, vol.21, no.11, pp. 4534 - 4543, 2012.

[12] D. Chen, and L. Cheng, "Spatially adapted total variation model to remove multiplicative noise.' image processing," IEEE Transactions on, vol. 21, no. 4, pp.1650-1662, 2012. 